19,11

\title{
Тепловые и магнитокалорические свойства манганитов $\mathrm{La}_{0.7} \mathrm{Sr}_{0.3-x} \mathrm{Ba}_{x} \mathrm{MnO}_{3}$
}

\author{
(C) А.Б. Гаджиев ${ }^{1}$, А.Г. Гамзатов ${ }^{1}$, А.Б. Батдалов ${ }^{1}$, 3.А. Хуршилова ${ }^{2}$, А.М. Алиев $^{1}$ \\ ${ }^{1}$ Институт фризики им. Х.И. Амирханова ДФИЦ РАН, \\ Махачкала, Россия \\ 2 Дагестанский государственный медицинский университет, \\ Махачкала, Россия \\ E-mail: mr.gadzhiev.93@mail.ru, gamzatov_adler@mail.ru
}

Поступила в Редакцию 8 июля 2021 г.

В окончательной редакции 13 июля 2021 г.

Принята к публикации 16 июля 2021 г.

Приведены результаты исследования влияния частичного замещения ионов $\mathrm{Sr}^{2+}$ ионами $\mathrm{Ba}^{2+}$ на теплофизические и магнитокалорические свойства манганита $\mathrm{La}_{0.7} \mathrm{Sr}_{0.3-x} \mathrm{Ba}_{x} \mathrm{MnO}_{3}(x=0 ; 0.02 ; 0.05$ и 0.10$)$ в интервале температур $100-400 \mathrm{~K}$ и в магнитном поле до $1.8 \mathrm{~T}$. Показано, что такое замещение приводит к значительному уменьшению $T_{C}$. В поведении температуропроводности $\eta(T)$ и теплопроводности $\kappa(T)$ вблизи $T_{C}$ обнаружены минимумы, которые связаны как с рассеянием фононов на локальных искажениях кристаллической решетки, так и на спиновых флуктуациях. Абсолютные значения теплопроводности убывают с ростом параметра беспорядка. Магнитокалорический эффект проявляет слабую зависимость от уровня замещения $(x)$.

Ключевые слова: Магнитокалорический эффект, манганиты, фазовые переходы, теплоемкость температурапроводность, теплопроводность.

DOI: $10.21883 /$ FTT.2021.12.51661.28s

\section{1. Введение}

Допированные манганиты $\left(\operatorname{Re}_{1-x} A_{x} \mathrm{MnO}_{3}\right.$, где $R e-$ редкоземельный элемент, $A$ - щелочноземельный металл), получившие широкую известность благодаря обнаруженному в них эффекту колоссального магнитосопротивления $[1,2]$ все еще остаются в поле зрения исследователей в том числе из-за наблюдавшего в них магнитокалорического эффекта, сравнимого по величине с эффектом в лучших магнитокалорических материалах $[3,4]$. Магнитокалорический эффект представляет интерес как основа для создания технологии магнитного охлаждения, которой в последнее время уделяется значительное внимание [4]. Кроме того, манганиты являются модельными объектами для исследования фундаментальных свойств сильнокоррелированых электронных систем.

Свойства манганитов в основном определяются соотношением количества разновалентных ионов $\mathrm{Mn}^{3+} / \mathrm{Mn}^{4+}$, а так же важную роль в поведении теплофизических параметров играют такие микроскопические параметры как локальные искажения кристаллической решетки, характеризуемые параметром беспорядка $\sigma^{2}=\Sigma x_{i} r_{i}^{2}-\left\langle r_{A}^{2}\right\rangle$ вызванные различаем в ионных радиусах и среднее значение ионных радиусов $A$-катионов $\left\langle r_{A}\right\rangle=0.7 r_{\mathrm{La}}+(0.3-x) r_{\mathrm{Sr}}+x r_{\mathrm{Ba}}$, где $r_{\mathrm{La}}, r_{\mathrm{Sr}}$ и $r_{\mathrm{Ba}}$ радиусы ионов $\mathrm{La}, \mathrm{Sr}$ и Ва соответвенно.

Корреляция между изменениями $\sigma^{2}$ и $\left\langle r_{A}\right\rangle$ и теплофизическими свойствами обнаружена при исследовании манганита $\operatorname{Pr}_{0.7} \mathrm{Sr}_{0.3-x} \mathrm{Ca}_{x} \mathrm{MnO}_{3}[5,6]$. Кроме того, было показано, что частичное замещение как в $A$ подрешетке, так и в подрешетке Mn может быть использовано для улучшения магнитокалорических свойств [5-7]. В данной композиции ион $\mathrm{Sr}^{2+}$ замещается на ион меньшего радиуса $\mathrm{Ca}^{2+}$, что создает „химическое давление“, приводящее к деформации (сжатию) кристаллической решетки и уменьшению как $\sigma^{2}$ так и $\left\langle r_{A}\right\rangle$. Следствием такого замещения является ослабление обменных взаимодействий и как следствие уменьшение $T_{C}$. В данной работе рассматривается противоположная ситуация: ион меньшего радиуса $\mathrm{Sr}^{2+}$ замещается на ион большего радиуса $\mathrm{Ba}^{2+}$, что должно привести к расширению кристаллической решетки и соответствующим изменениям в значениях $\sigma^{2}$, и $\left\langle r_{A}\right\rangle$. При расчетах брали значения ионных радиусов, соответствующие значению координационного числу 8: $r_{\mathrm{La}}=1.18 \AA, r_{\mathrm{Sr}}=1.26 \AA$, $r_{\mathrm{Ba}}=1.42 \AA[8]$.

В настоящей работе приводятся результаты экспериментального исследования влияния частичного замещения $\mathrm{Sr}$ на Ва на теплоемкость, теплопроводность, температуропроводность и магнитокалорический эффект в системе $\mathrm{La}_{0.7} \mathrm{Sr}_{0.3-x} \mathrm{Ba}_{x} \mathrm{MnO}_{3}(x=0 ; 0.02 ; 0.05$ и 0.10$)$ в интервале температур $100-400 \mathrm{~K}$ и в магнитных полях до $1.8 \mathrm{~T}$.

\section{2. Образцы и эксперимент}

Образцы были изготовлены по стандартной керамической технологии [9]. Теплоёмкость и температуропро- 
водность измерялись методом ас-калориметрии и эти данные использовались для определения теплопроводности, которые связаны соотношением $\kappa=\frac{d}{M} C_{P} \eta$, где $d$ - плотность образца, $M$ - его молярная масса, $C_{P}$ - теплоемкость, $\eta$ - температуропроводность, $\kappa-$ теплопроводность. Прямые измерения адиабатического изменения температуры $\Delta T_{\text {ad }}$ при изменении магнитного поля проводились модуляционным методом [10]. Суть метода заключается в том, что к образцу прикладывается переменное магнитное поле, которое благодаря магнитокалорическому эффекту индуцирует периодическое изменение температуры образца. Это изменение температуры регистрируется синхронным детектором посредством дифференциальной термопары, один спай которой приклеен к исследуемому образцу, другой - к медному блоку. Частота изменения переменного магнитного поля в данном эксперименте составляла $0.2 \mathrm{~Hz}$. Данная методика позволяет регистрировать изменение температуры с точностью не хуже $10^{-3} \mathrm{~K}$. Переменное магнитное поле напряженностью $1.8 \mathrm{~T}$ создавалось источником магнитного поля производства фирмы АМТиС. В качестве термодатчиков использовались медь-константановые и хромель-константановые термопары.

\section{3. Результаты и обсуждения}

Для установления связи между физическими свойствами и микроскопическими параметрами $\sigma^{2}$ и $\left\langle r_{A}\right\rangle$ были рассчитаны значения последних в зависимости от $x$, которые приведены в таблице, температура Кюри определена по максимуму аномалии теплоемкости.

Из таблицы видно, что замещение иона меньшего радиуса $(\mathrm{Sr})$ на ион большего радиуса $(\mathrm{Ba})$ приводит к росту среднего ионного радиуса $\left\langle r_{A}\right\rangle$ и параметра беспорядка $\sigma^{2}$. Следует отметить, что $\left\langle r_{A}\right\rangle$ и $\sigma^{2}$ поразному сказываются на магнитные и теплофизические свойства манганита. Рост среднего ионного радиуса сопровождается увеличением угла $\mathrm{Mn}-\mathrm{O}-\mathrm{Mn}$, что приводит к росту $T_{C}$ - это означает усиление интенсивности обменных взаимодействий. В то же время, искажение кристаллической решетки вызванное разбросом в ионных радиусах $A$-катионов, которые ассоциируются с $\sigma^{2}$, в данном случае вызывают увеличение объема элементарной ячейки и удлинению длин связей $\mathrm{Mn}-\mathrm{O}$ [11-13], что должно привести к ослаблению обменных взаимодействий и уменьшению $T_{C}$. В общем

Некоторые параметры системы $\mathrm{La}_{0.7} \mathrm{Sr}_{0.3-x} \mathrm{Ba}_{x} \mathrm{MnO}_{3}$

\begin{tabular}{c|c|c|c}
\hline$x$ & $T_{C}, \mathrm{~K}$ & $\left\langle r_{A}\right\rangle, \AA$ & $\sigma^{2}, \AA^{2}$ \\
\hline 0 & 362 & 1.2040 & $1.2 \cdot 10^{-3}$ \\
\hline 0.02 & 343 & 1.2072 & $2.1 \cdot 10^{-3}$ \\
\hline 0.05 & 324 & 1.2120 & $2.9 \cdot 10^{-3}$ \\
\hline 0.10 & 280 & 1.2200 & $5.3 \cdot 10^{-3}$
\end{tabular}

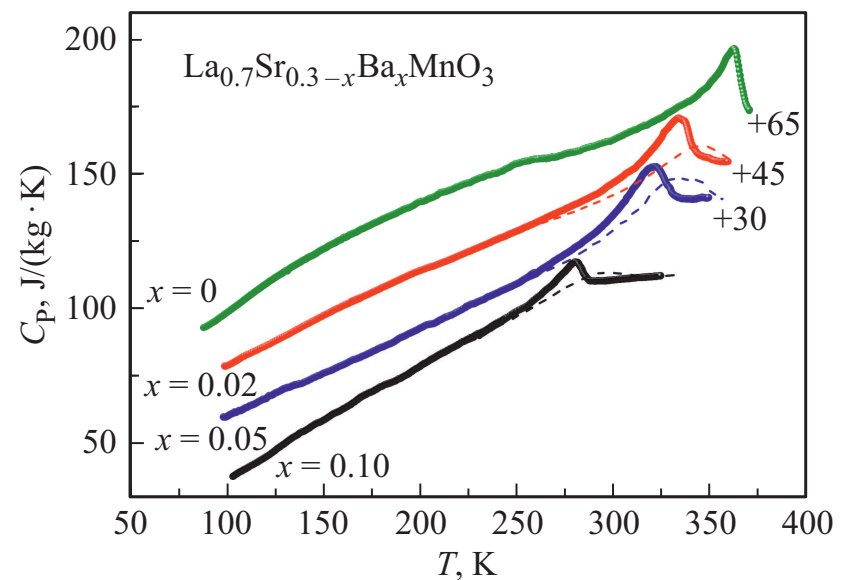

Рис. 1. Температурная зависимость теплоемкости для $\mathrm{La}_{0.7} \mathrm{Sr}_{0.3-x} \mathrm{Ba}_{x} \mathrm{MnO}_{3}$ при $H=0$ (точки) и при $H=1.8 \mathrm{~T}$ (пунктирные линии). Для наглядности кривые $C_{P}$ смещены на указанные единицы $C_{P}$.

случае, когда при замещении меняется и $\left\langle r_{A}\right\rangle$ и $\sigma^{2}$ зависимость $T_{C}=f\left(\left\langle r_{A}\right\rangle, \sigma^{2}\right)$ имеет сложный характер. Можно предположить, что наблюдаемое уменьшение величины $T_{C}$ с ростом $x$ связано с превалированием влияния параметра беспорядка $\sigma^{2}$ над ростом среднего ионного радиуса.

Рассмотрим более подробно, как такое замещение сказывается на поведении таких макроскопических параметров, как теплоёмкость, температуропроводность, теплопроводность и магнитокалорический эффект. На рис. 1 представлены результаты измерения температурной зависимости теплоемкости для системы $\mathrm{La}_{0.7} \mathrm{Sr}_{0.3-x} \mathrm{Ba}_{x} \mathrm{MnO}_{3}$ при $H=0$ и $H=1.8 \mathrm{~T}$. Для всех образцов наблюдается ярко выраженные аномалии, связанные с фазовым переходом ферромагнетикпарамагнетик (ФМ-ПМ), с максимумом при температу$\operatorname{pax} T_{C}=362,343,324$ и $280 \mathrm{~K}$ для $x=0,0.02,0.05$ и 0.1 соответственно. Как видим, из рисунка, магнитное поле подавляет аномалии и смещает температуру максимума в сторону высоких температур. Увеличение концентрации Ва приводит к смещению $T_{C}$ в сторону низких температур. Связано это с уменьшением обменного взаимодействия между ионами марганца вследствие увеличения расстояния между магнитоактивными атомами при замещении ионов стронция ионами бария с большим ионным радиусом.

Из данных теплоемкости были получены температурные зависимости аномальной части теплоемкости и изменения энтропии фазового перехода при $H=0$, которые приведены на рис. 2, $a$ и $b$. Аномальная часть теплоемкости определялась как $\Delta C_{P}(T)=C_{P}-C_{\mathrm{ph}}$, где $C_{\mathrm{ph}}-$ решеточный вклад, полученный путем экстраполяции дебаевской кривой теплоемкости, $C_{P}-$ измеренная величина теплоемкости. Как видно из рис. 2, $a$, максимальное значение скачка теплоемкости в области фазового перехода составляет $\Delta C_{P} \approx 28 \mathrm{~J} / \mathrm{mol} \cdot \mathrm{K}$ для образца с $x=0$ 

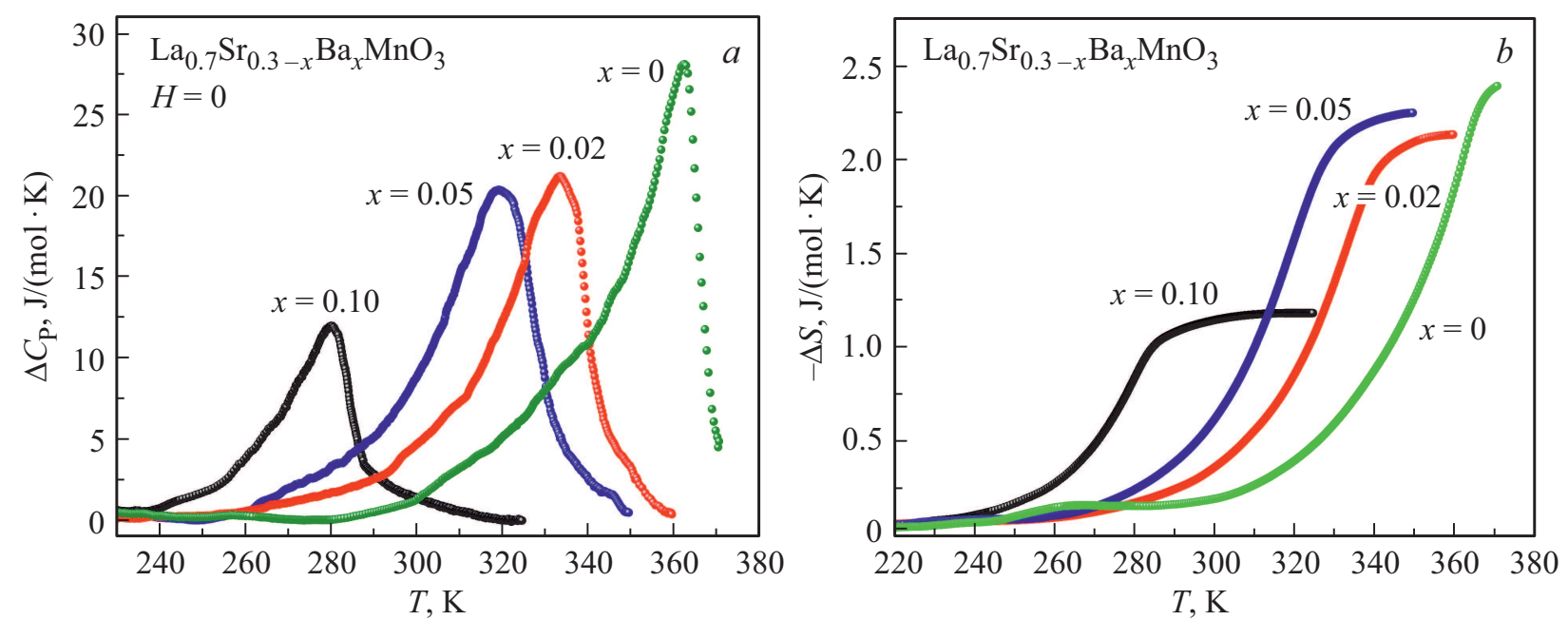

Рис. 2. $a)$ Температурная зависимость аномальной части теплоемкости $(a)$ и изменения энтропии фазового перехода при $H=0(b)$.
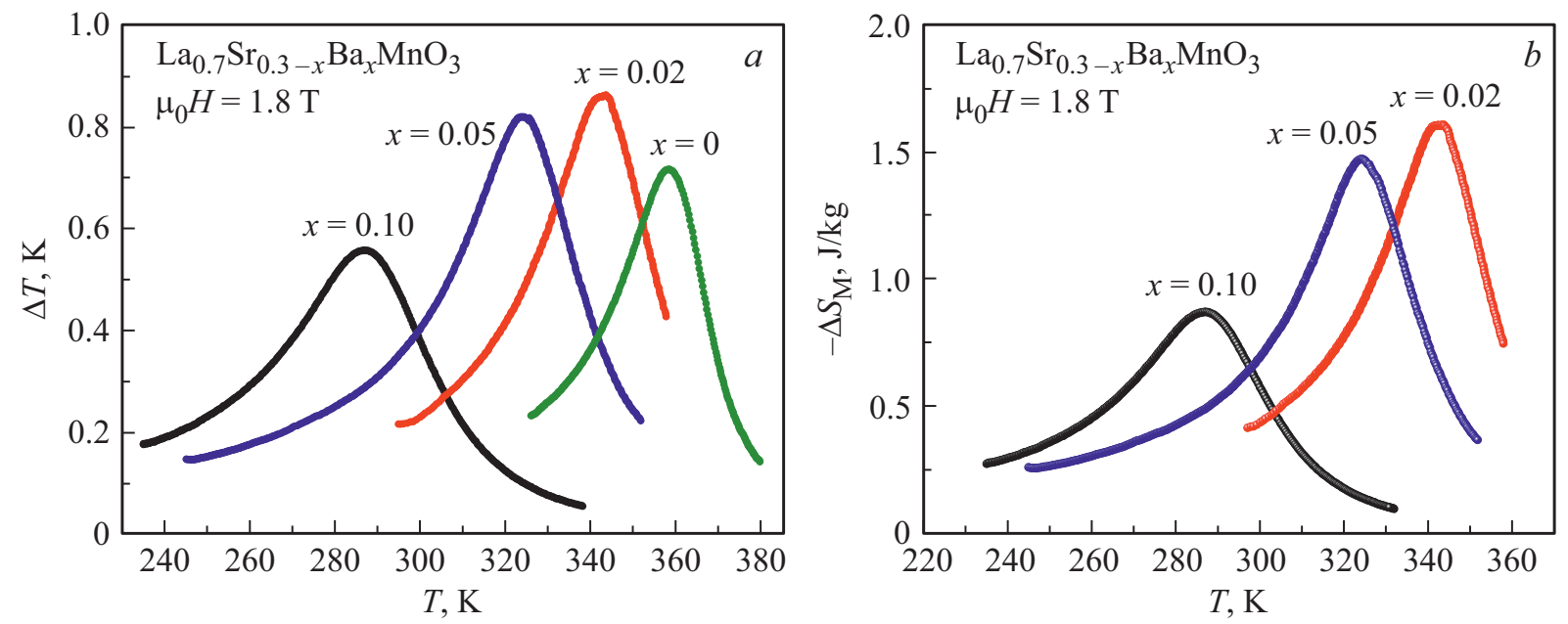

Рис. 3. Температурная зависимость МКЭ $(a)$ и изменения магнитной части энтропии $\Delta S_{M}$ в магнитном поле $1.8 \mathrm{~T}(b)$.

и минимальное значение $\Delta C_{P} \approx 12 \mathrm{~J} / \mathrm{mol} \cdot \mathrm{K}$ наблюдается для образца с $x=0.1$, то есть с ростом замещения величины скачка $\Delta C_{P}$ значительно уменьшается.

На рис. 2,b приведены температурные зависимости изменения энтропии, связанные с разупорядочением магнитной системы при фазовом переходе ферромагнетик-парамагнетик, которые определялись по формуле: $\Delta S^{*}(T)=\int\left(\Delta C_{P} / T\right) d T$. Значения $\Delta S^{*}$ для системы $\mathrm{La}_{0.7} \mathrm{Sr}_{0.3-x} \mathrm{Ba}_{x} \mathrm{MnO}_{3}$ изменяются от 1.20 до $2.38 \mathrm{~J} / \mathrm{K}$, что значительно меньше идеальных значений для модели Изинга $\left(\Delta S^{*}=R \ln 2=5.7 \mathrm{~J} / \mathrm{K}\right)$ и Гейзенберга $\left(\Delta S^{*}=R \ln 4=11.52 \mathrm{~J} / \mathrm{K}\right)[10]$. Такого рода расхождения экспериментальных данных и теоретических оценок $\Delta S^{*}$ характерны для манганитов многократно обсуждались в литературе [7,15-17].

На рис. 3, $а$ приведена температурная зависимость МКЭ для $\mathrm{La}_{0.7} \mathrm{Sr}_{0.3-x} \mathrm{Ba}_{x} \mathrm{MnO}_{3}$ в магнитном поле $1.8 \mathrm{~T}$. Как видим из рисунка с ростом концентрации Ва температура максимума эффекта смещается в сторону низких температур, что хорошо согласуется с данными теплоемкости (рис. 1), и расчетами микроскопических параметров. Максимальная величина МКЭ в поле $1.8 \mathrm{~T}$ равна $\Delta T_{\mathrm{ad}}=0.86 \mathrm{~K}$ и наблюдается для образца $\mathrm{La}_{0.7} \mathrm{Sr}_{0.28} \mathrm{Ba}_{0.02} \mathrm{MnO}_{3}$ при $T=343 \mathrm{~K}$. Следует отметить, для образцов с $x=0,0.02$ и 0.05 величина МКЭ слабо меняется с допированием и колеблется в пределах $\Delta T_{\mathrm{ad}} \sim 0.8 \mathrm{~K}$. При этом $T_{C}$ смещается более чем на $40 \mathrm{~K}$. Это означает, что путем регулирования уровня замещения можно получить нужную $T_{C}$ в широкой области температур, при этом слабо меняя величину МКЭ, что является важным преимуществом для магнитокалорических материалов.

На рис. 3, $b$ приведены результаты исследования температурной зависимости изменения магнитной части энтропии, которые были получены из данных по теплоемкости в поле и прямых измерений МКЭ с помощью 

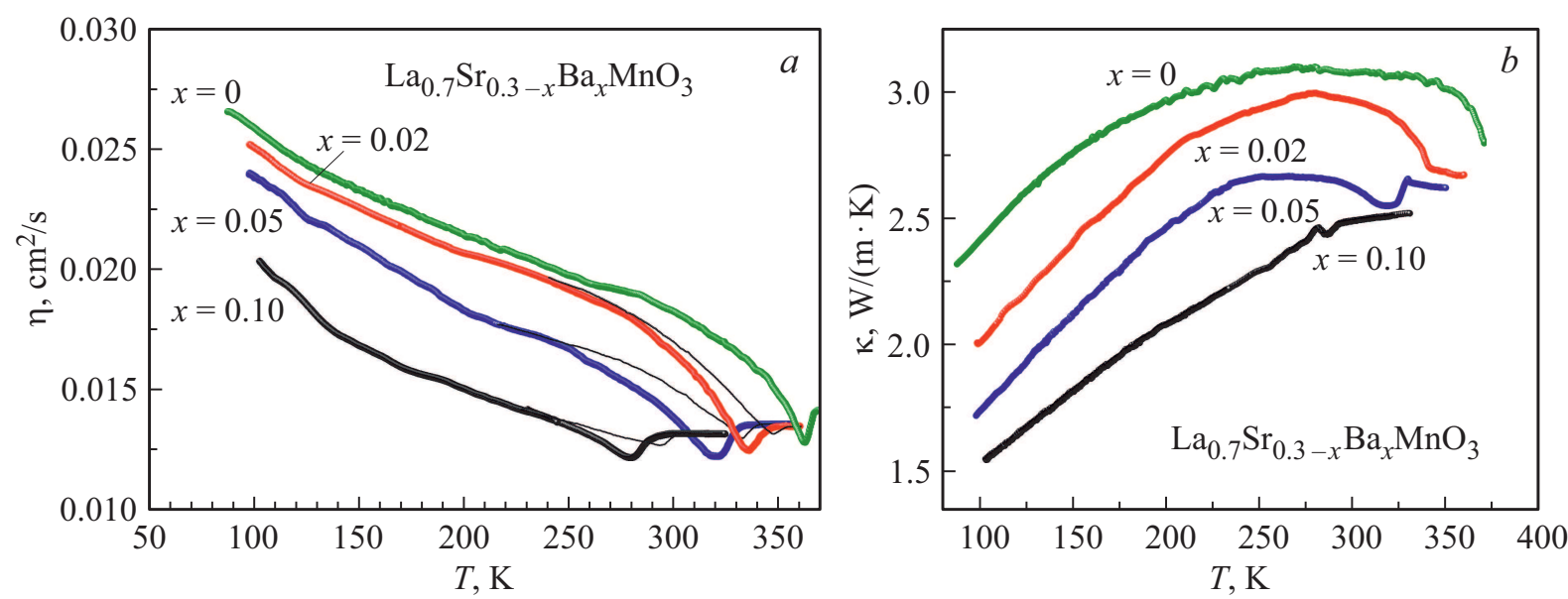

Рис. 4. Температурные зависимости температуропроводности при $H=0$ и $H=1.8 \mathrm{~T}(a)$ и теплопроводности (b) для $\mathrm{La}_{0.7} \mathrm{Sr}_{0.3-x} \mathrm{Ba}_{x} \mathrm{MnO}_{3}$ при $H=0$.

формулы $\Delta S_{M}=\Delta T_{\mathrm{ad}} C_{P}(T, H) / T$, где $\Delta T_{\mathrm{ad}}-$ экспериментальные данные прямых измерений, $C_{P}(T, H)-$ температурная зависимость теплоемкости в магнитном поле $1.8 \mathrm{~T}[18]$. Полученные значения $\Delta T_{\text {ad }}$ и $\Delta S_{m}$ находятся в пределах, приведённых в литературе для других манганитов [3,5].

Рассмотрим более подробно механизмы теплопереноса. Многочисленные экспериментальные работы показали, что фононный механизм теплопередачи является доминирующим в манганитах [19-21]. В таком случае для анализа механизмов рассеяния фононов можно воспользоваться данными по температуропроводности. Температуропроводность характеризует скорость изменения температуры в нестационарных тепловых процессах и связана с длиной свободного пробега фононов $l_{\mathrm{ph}}$ соотношением $\eta=\frac{1}{3} l_{\mathrm{ph}} v_{s}$, где $v_{s}-$ скорость распространения звука. Предполагая, что $v_{s}$ слабо зависит от температуры по измерениям $\eta(T)$ можно проследить за ходом изменения $l_{\mathrm{ph}}(T)$ и делать соответствующие выводы о механизме рассеивания фононов. На зависимости $\eta(T)$ (рис. 4,a) для всех образцов близи $T_{C}$ наблюдаются минимумы, которые в магнитном поле сглаживаются. Такие аномалии уже наблюдались для ряда манганитов и связывались с рассеянием фононов на локальных искажениях кристаллической решетки, вызванных эффектом Яна-Теллера [19,21]. Вид минимумов и их глубина, в зависимости от концентрации Ва и магнитного поля говорят о том, что при интерпретации результатов по теплопереносу необходимо рассматривать несколько механизмов, которые приводят к изменению скорости рассеяния носителей тепла.

В случае рассеяния фононов только на искажениях Яна-Теллера на зависимости $\eta(T)$ наблюдался бы только излом без минимума. Вид аномалий на зависимости $\eta(T)$ вблизи $T_{C}$ говорит о том, что кроме искажений Яна-Теллера необходимо рассматривать и другие механизмы рассеяния фононов. Из рисунка видно, что глубина минимума растет по мере уменьшения искажений кристаллической структуры $\left(\sigma^{2}\right)$ и усиления магнитных флуктуации, на основании чего можно предположить, что имеет место и рассеяние фононов на спиновых флуктуациях. Пригодность такой модели для объяснения поведения $\kappa(T)$ вблизи $T_{C}$ была продемонстрирована экспериментально при исследовании теплопроводности манганита $\left(\mathrm{La}_{1-x} \mathrm{~N}_{d x}\right)_{0.7} \mathrm{~Pb}_{0.3} \mathrm{Ca}_{x} \mathrm{MnO}_{3}$ [22], а теоретически была обоснована в работа [23]

Зависимость $\kappa(T)$ приведена на рисунке $4(b)$. Видно, что абсолютная величина теплопроводности растет по мере уменьшения параметра беспорядка $\left(\sigma^{2}\right)$ и имеет характерный для манганитов температурный ход [19,21]. Переход в ферромагнитную фазу сопровождается ростом величины $\kappa$. Уменьшение $\kappa$ с ростом $\sigma^{2}$ указывает на значительную роль локальных искажений в ограничении фононного потока тепла.

\section{4. Заключение}

Проведено исследования влияния замещения иона $\mathrm{Sr}^{2+}$ на ион $\mathrm{Ba}^{3+}$ на магнитные, тепловые и магнитокалорические свойства манганита $\mathrm{La}_{0.7} \mathrm{Sr}_{0.3-x} \mathrm{Ba}_{x} \mathrm{MnO}_{3}$ $(x=0 ; 0.02 ; 0.05 ; 0.10)$. Показано, что такое замещение проводит к росту как параметра беспорядка $\sigma^{2}$ так и среднего ионного радиуса $A$-катионов $\left\langle r_{A}\right\rangle$, и ослаблению интенсивности обменных взаимодействий. Аномальная часть теплоемкости и изменения энтропии фазового перехода убывает с ростом концентрации Ва. Теплопроводность носит преимущественно фононный характер и убывает по абсолютной величине по мере роста параметра беспорядка $\sigma^{2}$.

Для объяснения наблюдаемых аномалий в поведении температурапроводности и теплопроводности при $T_{C}$ необходимо привлечь как рассеяние фононов на локальных искажениях кристаллической решетки, вызванные эффектом Яна-Теллера, так и взаимодействие фононов со спиновыми флуктуациями. Для исследования МКЭ 
применялся метод модуляции магнитного поля. Полученные данные указывают на слабую зависимость эффекта от уровня замещения, что представляет определенный практический интерес.

\section{Благодарности}

Авторы выражают благодарность S.-C. Yu за предоставленные для исследования образцы.

\section{Финансирование работы}

Работа выполнена при частичной финансовой поддержке гранта РФФИ № 20-58-54006.

\section{Конфликт интересов}

Авторы не имеют конфликтов интересов.

\section{Список литературы}

[1] V. Markovich, A. Wisniewski, H. Szymczak. Handb. Magn. Mater. 22, 1-201 (2014).

[2] E. Dagotto, T. Hott, A. Moreo. Phys. Rep. 344, 1 (2001).

[3] M.F. Phan, S.C Yu. J. Magn. Magn. Mater. 308, 325 (2007).

[4] V. Franco, J.-S. Blazquez, J.J. Ipus, J.Y. Low, L.M. MarenoRamiros, A. Conde. Prog. Mater. Sci. 93, 112-232 (2018).

[5] A.B. Batdalov, A.G. Gamzatov, A.M. Aliev, N. Abdulkadirova, P.D.H. Yen, T.D. Thanh, N.T. Dung, S.-C. Yu. J. Alloys Comp. 782, 729 (2019).

[6] A.G. Gamzatov, A.M. Aliev, P.D.H. Yen, K.X. Hau, Kh.E. Kamaludinova, T.D. Thanh, N.T. Dung, S.-C. Yu. J. Magn. Magn. Mater. 474, 477-481 (2019).
[7] A.G. Gamzatov, A.B. Batdalov, A.M. Aliev, Z. Khurshilova, M. Ellouze, F. Ben Jemma. J. Magn. Magn. Mater. 443, 352 (2017).

[8] J.L. Cohn. J. Supercond. 13, 291 (2000).

[9] А.Б. Гаджиев, А.Г. Гамзатов, А.Б. Батдалов, А.М. Алиев, Д. Нанто, Б. Курниаван, С.-Ч. Юу, Д.Х. Ким. Челябинский физ.-мат. журн. 6, 1, 87-94 (2021).

[10] А.М. Алиева, А.Б. Батдалова, В.С. Калитка. Письма в ЖЭТФ 90, 10, 736 (2009).

[11] C. Martin, A. Maignan, M. Hervieu, B. Raveau. Phys. Rev. 60, 12190 (1999).

[12] А.И. Абрамович, Л.И. Королева, А.В. Мичурин, О.Ю. Горбенко, А.Р. Кауль, М.Х. Машаев, Р. Шимчак, Б. Кжиманска. ФТТ 44, 5, 888 (2002).

[13] Lide M. Rodriges, J. Paul. Attfild. Phys. Rev. 54, 15622 (1996).

[14] Y. Moritomo, A. Machida, E. Nishibori, M. Takata, M. Sakata. Phys. Rev. 64, 214409 (2001).

[15] A.G. Gamzatov, A.M. Aliev, A.B. Batdalov, H. Ahmadvand, H. Salamati, P. Kameli. J. Mater. Sci. 49, 294 (2014).

[16] М.Н. Хлопкин, Г.Х. Панова, А.А. Шиков, В.Ф. Синянский, Д.А. Шулятев. ФТТ 42, 111 (2000).

[17] А.Г. Гамзатов, А.Б. Батдалов, А.М. Алиев, М. Ellouze, F. Jemma. ФTT 59, 10 (2017).

[18] Vitalij K. Pecharsky, Karl A. Gschneinder Jr. J. Magn. Magn. Mater 200, 44 (1999).

[19] J.L. Cohn, J.J. Neumeier, C.P. Popoviciu, K.J. McClellan, Th. Leventouri. Phys. Rev. 56, 8495 (1997).

[20] D.W. Visser, A.P. Ramírez, M.A. Subramanian. Phys. Rev. Lett. 78, 3947 (1997).

[21] A.M. Aliev, A.B. Batdalov, A.G. Gamzatov. Low Temp. Phys. 36, 171 (2010).

[22] M. Tachibana, E. Takayama-Muromachi. Appl. Phys. Lett. 92, 2507 (2008).

[23] K. Kawasaki. Prog. Theor. Phys. 29, 801 (1963).

Редактор К.В. Емцев

\section{Продолжение публикащии материалов семинара в ФТТ № 01/22}

